\title{
Characterization of Biogenic Nanoparticles Via In-Situ Correlative Secondary Electron Helium Microscopy and Secondary Ion Mass Spectrometry
}

\author{
Christelle Guillermier ${ }^{1 *}$, David Medina Cruz ${ }^{2}$, Jean-Nicolas Audinot ${ }^{3}$ and Tom Wirtz ${ }^{3}$ \\ ${ }^{1}$ Carl Zeiss SMT, Inc., One Corporation Way, Peabody, MA, USA. \\ ${ }^{2}$ Nanomedicine Science and Technology Center, Northeastern University, Boston, MA, USA. \\ ${ }^{3}$ Advanced Instrumentation for Ion Nano-Analytics, Department of Materials Research and Technology, \\ Luxembourg Institute of Science and Technology (LIST), Belvaux, Luxembourg. \\ * Corresponding author: christelle.guillermier@zeiss.com
}

Over the past several years, the use of both nanoparticles and nanostructured surfaces have emerged as alternatives to the use of antibiotics. Indeed, nanoparticles decrease bacterial survival without being highly toxic to mammalian cells. These nanoparticles can be composed of a variety of materials including silver, gold, titanium dioxide, zinc oxide, silica, gold-dendrimer nanocomposites, and carbon. More recently, an environmentally safe approach was developed for synthetizing Se nanoparticles in the size range of 90$150 \mathrm{~nm}$ using E. coli, Pseudomonas aeruginosa, Methicillin resistant S. aureus [1].

Transmission electron microscopy (TEM) and energy dispersive X-ray spectroscopy (EDX) are the analytical techniques of choice to determine the nanoparticles' morphology and chemical composition. Although sensitive enough to detect trace elements, standard EDX cannot provide chemical information at sub-micrometric spatial resolution.

Here we demonstrate the analytical capabilities of the Helium Ion Microscope (ZEISS ORION NanoFab) recently equipped with a Secondary Ion Mass Spectrometer (SIMS) for the characterization of biogenic nanoparticles. The ORION NanoFab is an ion microscope that allows for high resolution secondary electron (SE) imaging with a $\mathrm{He}^{+}$beam and nanofabrication (sputtering/beam assisted etching or deposition) with $\mathrm{Ne}^{+}$, with focused probe sizes of $0.5 \mathrm{~nm}$ and $2 \mathrm{~nm}$, respectively [2]. With the addition of the SIMS detector, this instrument can now provide, in-situ chemical information at an unprecedented $\sim 15$ $\mathrm{nm}$ of spatial resolution along with the correlative, high resolution SE image of the exact same area [3-7]. The new SIMS is a double focusing magnetic sector mass spectrometer equipped with four movable Channel Electron Multipliers (CEMs) positioned on the same focal plane. This setup allows for the simultaneous detection of up to 4 different masses (all elements and additional small molecular fragments) sputtered from the exact same probe-sample interaction nanovolume. The accessible mass range is $\mathrm{M}_{\max } / \mathrm{M}_{\min }=100$ with a maximum mass detection up to $250 \mathrm{amu}$.

In this study we focused on the characterization of nanoparticles synthetized utilizing E. coli and Staphylococcus aureus along with their Multidrug-Resistant strain analogs. Bacteria were cultured and incubated with the addition of $\mathrm{Na}_{2} \mathrm{SeO}_{3}$ salt to the media for $24 \mathrm{hrs}$. The solution was then purified via centrifugation, isolating the newly synthetized nanoparticles. The product of the centrifugation was resuspended in water, and a drop of the solution was deposited on a silicon chips and let to air dry.

Figure 1. shows the results of SIMS analysis with the NanoFab. The SE image shows the presence of nanoparticles of various size along with organic debris. Also shown is the mass separated images of oxygen, $\mathrm{CN}^{-}$, and selenium. The $\mathrm{CN}^{-}$signal shows the location of the organic debris while the dispersion of the selenium nanoparticles is also clearly visible. The correlation of the Selenium-SIMS and secondary 
electron images evince the presence of Se in the newly synthetized nanoparticles as shown by the spatial relationship between the two.

References:

[1] Medina Cruz, David, Gujie Mi, and Thomas J. Webster. Journal of Biomedical Materials Research Part A106.5 (2018), 1400-1412.

[2] G. Hlawacek, A. Gölzhäuser, Helium Ion Microscopy, Springer, 2017.

[3] T. Wirtz, P. Philipp, J.-N. Audinot, D. Dowsett, S. Eswara, Nanotechnology 26 (2015), 434001.

[4] T. Wirtz, D. Dowsett and P. Philipp in "SIMS on the Helium Ion Microscope: A Powerful Tool for High-Resolution High-Sensitivity Nano-Analytics”. In: Hlawacek G., Gölzhäuser A. (eds) Helium Ion Microscopy (Springer, Cham) 297.

[5] D. Dowsett, T. Wirtz, Anal. Chem. 89 (2017), 8957.

[6] T. Wirtz, O. De Castro, J.-N. Audinot, and P. Philipp, Annual Review of Analytical Chemistry 12 (2019) [5] P. Gratia, G. Grancini, J.-N. Audinot, X. Jeanbourquin, E. Mosconi, I. Zimmermann, D.

Dowsett, Y. Lee, M. Grätzel, F. De Angelis, K.Sivula, T. Wirtz, M. K. Nazeeruddin, J. Am. Chem. Soc. 138 (2016), 15821

[7] F. Vollnhals, J.-N. Audinot, T. Wirtz, M. Mercier-Bonin, I. Fourquaux, B. Schroeppel, U. Kraushaar, V. LevRam, M. H. Ellisman, S. Eswara, Anal. Chem. 89 (2017), 10702-10710
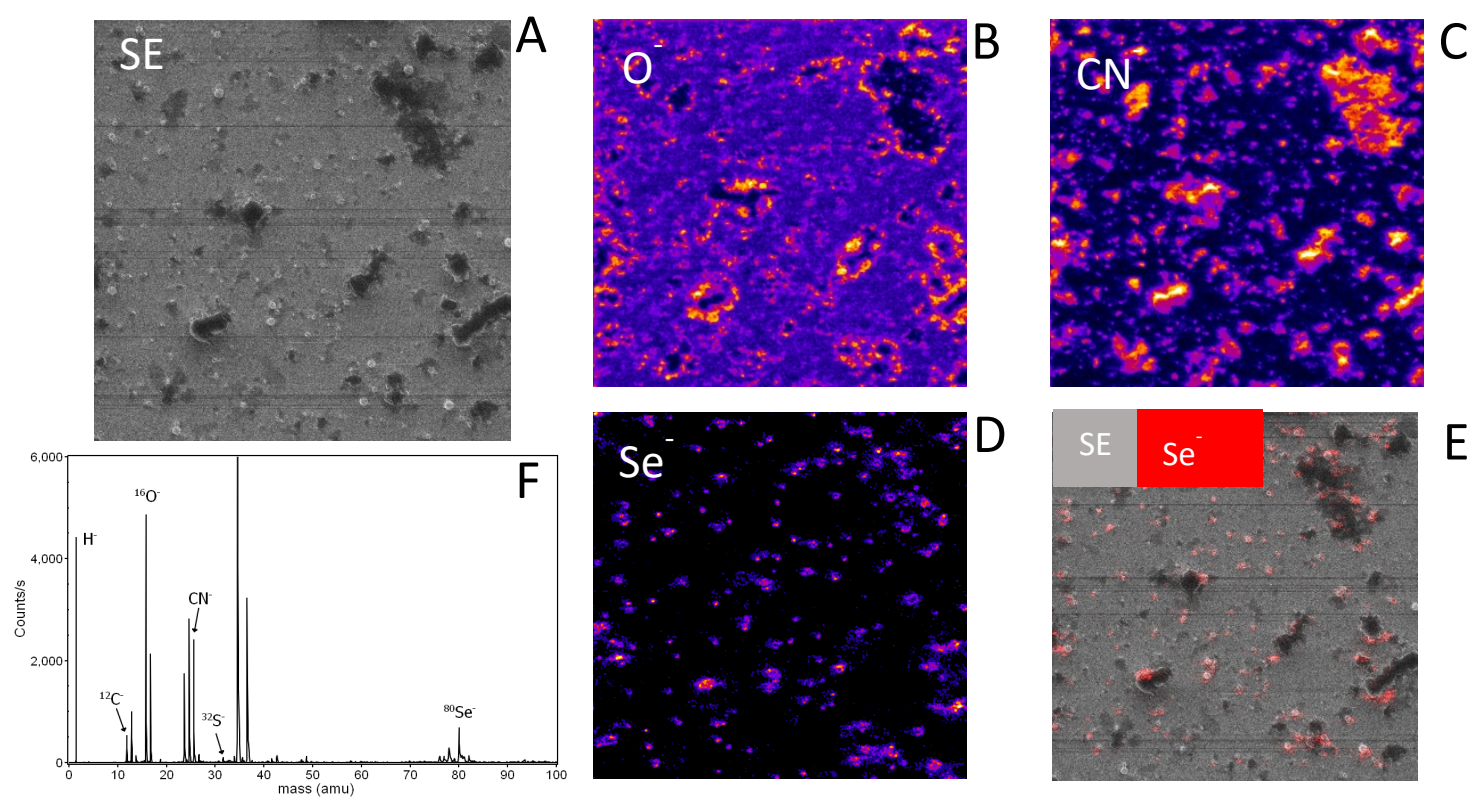

Figure 1. $10 \mu \mathrm{m} \times 10 \mu \mathrm{m}$ FoV images of NPs synthetized by Multidrug-Resistant E. Choli incubated with $\mathrm{Na}_{2} \mathrm{SeO}_{3}$. A: SE image obtained with the Helium Ion Microscope. B, C, D: mass separated images of $\mathrm{O}^{-}$, $\mathrm{CN}^{-}$and $\mathrm{Se}^{-}$. E: SE- Selenium correlative image. F: mass spectrum 